\title{
Medicine in the early twenty-first century: paradigm and anticipation - EPMA position paper 2016
}

\author{
Olga Golubnitschaja 1,2,3*, Babak Baban ${ }^{1,4,5}$, Giovanni Boniolo 1,6,7 Wei Wang 1,8,9,10,11, Rostyslav Bubnov 1,12,13, \\ Marko Kapalla ${ }^{1}$, Kurt Krapfenbauer ${ }^{1}$, Mahmood S. Mozaffari ${ }^{1,4,5}$ and Vincenzo Costigliola ${ }^{1,14}$
}

\begin{abstract}
Keywords: Traditional complementary alternative medicine, Person-centred medicine, Individualised medicine, Stratified medicine, Personalised medicine, Precision medicine, Predictive, preventive, and personalised medicine, Advantage, Limitation, Implementation
\end{abstract}

\section{Background}

Challenges of "standardisation" and "individualisation" have always been characteristic for medical services. In terms of individualisation, the best possible individual care is the ethical imperative of medicine, and it is a good right of any patient to receive it. However, in terms of standardisation, all the available treatments are based on guideline recommendations derived from large multicentre trials with many thousands of patients involved. In the most optimal way, the standardisation and individualisation should go hand-in-hand, in order to identify the right patient treating him/her with the right medication and the right dose at the right time point!

Further, in paradigm and anticipation, there is a big discrepancy between "disease care" and "health care" which dramatically impacts ethical and economical aspects of medical services.

Several approaches have been suggested in ancient and modern medicine to conduct medical services in a possibly optimal way. What is the difference amongst all of them and how big is the potential beyond corresponding approach to satisfy the needs of the individual, the patient, professional groups involved and society at large?

On behalf of the "European Association for Predictive, Preventive and Personalised Medicine," the dedicated

\footnotetext{
* Correspondence: Olga.Golubnitschaja@ukb-uni-bonn.de

${ }^{1}$ European Association for Predictive, Preventive and Personalised

Medicine, Brussels, Belgium

${ }^{2}$ Radiologic Department, Rheinische Friedrich-Wilhelms-University of Bonn,

Sigmund-Freud-Str. 25, 53105 Bonn, Germany

Full list of author information is available at the end of the article
}

EPMA working group provides a deep analysis in the issue followed by the expert recommendations considering the multifaceted aspects of both "disease care" and "health care" practices including ethics and economy, life quality of individuals and patients, interests of professional groups involved, benefits of subpopulations, health care system(s) and society as a whole.

\section{Traditional, complementary and alternative medicine (TCAM)}

TCAM, also called "integrative medicine," is considered as an amorphous concept comprising a range of ancient, long-standing but still evolving treatment approaches being practised mainly in their countries of origin as well as in countries into which corresponding expertise has been "imported" [1]. TCAM refers to health practices, approaches, knowledge and beliefs incorporating plant-, animal- and mineral-based medicines, spiritual therapies, manual techniques and exercise (e.g. in form of acupuncture, dietary therapy, herbal medicine, moxibuston, Taiji, Ayurveda, amongst others) applied singularly or in combination to diagnose, treat and prevent illnesses or maintain wellbeing [2]. However, the educational level of the doctor is critical for the quality of TCAM that depends on the national/local curricula varying substantially from country to country and, therefore, may not be adequate enough to fully realise potential benefits of various forms of TCAM modalities. TCAM approaches are frequently considered as being non-evidence based [3]. Further deficiencies arise from evident philosophical and religious differences as well as some cultural barriers 
between the countries of origin and countries into which TCAM is "imported" [1]. Nevertheless, in addition to the conventional medicine, TCAM is getting more and more popular and well-pursued in Western countries. From view point of predictive and preventive medicine, TCAM provides a unique expertise for recognising the so-called suboptimal health conditions before a clinical manifestation of severe pathologies [3-5]. These global trends make particularly attractive consideration regarding innovative hybrid approaches which would utilise advantages of both TCAM and modern medicine and, therefore, benefiting patients and enriching the spectrum of tools and overall expertise of the dedicated professional groups assuring the reproducibility of TCAM technologies and outcomes [6]. However, those approaches are currently underdeveloped and require additional major efforts in terms of multi-professional collaboration, scientific and technological discoveries and extensive financial support.

\section{Person-centred medicine}

The main idea of the person-centred medicine is to promote health and, therefore, reduce disease burden. In this concept, any health condition is considered as an individual state of physical, mental, social and spiritual well-being. Contextually, health care approaches are prioritised by person-centred medicine compared to a disease care. Humanistic interpretation of medicine is characteristic including the articulation of science, enhanced understanding of positive health versus illness, emphasised personalisation of all medical services as well as strong patient empowerment and essential responsibility of every person, at individual and community levels. "All for one and one for all" - a smart but perhaps a bit naive slogan introduced by the Three Musketeers fits well to the philosophy of the person-centred medicine. Therefore, a realisation of those ideas demands clear definitions and validated strategies to reach a reasonable level of maturity in health care [7].

\section{Individualised medicine}

A great strength of individualised medicine (IM) is to provide a holistic and integrative approach for medical care. IM comprises curative, rehabilitative and preventive examination as well as treatment methods customised for the individual and the patient [8]. IM well recognises a multidimensional interaction of internal and external risk factors, genetic background, age, gender, environmental risk factors, lifestyle, culture and beliefs as well as social status in the overall predisposition of individuals to specific diseases, the disease development, the natural course of disease and the response to therapeutic intervention. These factors vary from individual to individual. Contextually, IM aims to categorise patients into clinically relevant subgroups (that is the content of the "Stratified medicine" - see below). Hence, at the heart of the concept of IM is a stratification that "individualises" a one-size-fits-all standardised intervention into a group-specific intervention. Less clear concepts and approaches are provided by IM towards "predictive and preventive medicine"-see below.

\section{Stratified medicine}

Stratified medicine means looking at large groups of affected individuals (e.g. cancer patients) to try and find ways of predicting which treatments/patient sub-types are likely to respond to. Specifically in cancer, it involves looking in detail at the cancer cells and their genetic make-up. The purpose of the approach is to find out which treatment algorithms are more likely to work [9]. Patient stratification is one step towards individualised patient treatments and so-called "personalised medicine"see below.

\section{Personalised medicine}

The term "personalised medicine" is the keyword to refer to the best possible, most optimal and innovative medical approaches in the early twenty-first century, to justify grant applications and to receive dedicated budgets. However, in order to make anticipation by personalised medicine as realistic as possible, this term should be pragmatically sub-divided into its clear subcategories, namely "semi-personalised" versus "true personalised" as it has been discussed and published elsewhere in scientific literature [10].

"Semi-personalised medicine" compromises between standardisation and individualisation in medicine, the first step of which is the stratification of big patientgroups according to certain well-known characteristics (e.g. specific biological characteristics of the tumour). In the next step, individual patients within the group are treated according to the algorithms adapted to the entire stratified group. Consequently, the treatment efficacy varies from patient to patient within the group, since a limited number of characteristics in common is considered by the treatment algorithm; all other individual characteristics are not taken into account but may sufficiently impact individual outcomes.

"True personalised medicine" is based on the "individual patient profile" (see "Predictive, preventive and personalised medicine (PPPM)" section) directing to a tailored therapy that maximises the efficacy for that one patient in particular.

However, a disadvantage of "personalised medicine" is that its contents are adapted to the needs of disease care for treatments of diseased individuals and individual patient cohorts but not for health care of individuals to 
maintain in a good mental and physical shape avoiding clinical manifestation of diseases.

\section{Precision medicine}

The terms precision, personalised and individualised medicines are often used interchangeably [11]. Precision medicine is a concept of therapeutic and preventive modality for disease that takes into account individual variability in genes, environment and lifestyle. It refers to the tailoring of medical treatment to the individual characteristics of each patient [12]. Precision medicine is considered a relatively new approach in disease and health care, although it has been around for a while and has limited application in certain fields of medicine such as blood transfusion and organ transplantation. Further, precision medicine faces a number of serious challenges that need to be addressed [13-18] as summarised below.

a) Knowledge gap: Extensive and costly long-term education is required for all health care system authorities, physicians and participants to fully understand the dynamic and potential objectives of precision medicine.

b) Authority and interpretation: Even for the field specialists, an interpretation of DNA data for individual health outcomes remains sophisticated and the problem of interpretability continues to grow. Consequently, many doctors are simply not able to make sense of genetic tests and to communicate the results accurately to their patients.

c) Data storage: Gene sequencing of an individual produces massive amounts of data. The sequencing of thousands, if not millions, of people will produce unimaginable amount of data. How will we store the data and effectively analyse to derive useful information and to interpret the data?

d) Pathogenic mechanism: Many diseases have complex and multifactorial pathogenic mechanisms which would make it very difficult to identify a specific gene responsible for their manifestations.

e) Most technologies and equipment required for effective implementation of precision medicine are still in embryonic stages.

f) Privacy/security: Cyberattack is increasingly a major hurdle to maintaining privacy and security for all particularly given the current state of world affairs. Such valid concerns are already well-recognised for economic, energy and defence sectors across the globe. Precision medicine relies on massive public and personal data requiring sophisticated and extensive infrastructure and technology. Thus, vulnerability due to breach of security and privacy violation could have devastating consequences for the successful implementation of precision medicine
[19] and data misusing, e.g. for economic and political purposes with a consequent discrimination of affected individuals and even (sub)populations involved in the database containing sensitive genetic information and family history amongst others.

g) Coordination and policies: For precision medicine to have its greatest impact, federal and private health insurance companies have no option but to become comfortable with value-based drug pricing.

h) Variability of phenotypic features in population: It is difficult, if not impossible, to detect, decipher and utilise phenotypic characteristics of every individual as indicators for diseases as seems to be proposed by precision medicine.

i) Data relevance: The usefulness of data gathered from smaller groups may not be sufficient to make larger population health recommendations.

j) Culture: Prevention of abuse of information for unintended purposes such as screening potential partners and denying insurance coverage is a serious concern. How will this affect the culture? Will we be cultivating a different kind of racism, on a genetic basis?

k) Ownership: Who will have ownership of the data? Will it be the government? It is noteworthy that the FDA has blocked companies from allowing individuals to have access to their own genetic information. Will this change as part of the new initiative?

l) Compliance: There is no binding protocol to guarantee that all individuals would follow the recommendations made by precision medicine so the diseases could be prevented, controlled or cured.

m)Drug/device industry: Genetic research and development of treatment options have been very promising and productive in the private sector. How will government involvement affect research? Will governmental agencies work cooperatively with them or competitively?

n) Diversion from overarching goals of health care system: The focus and concentration of human and financial resources on precision medicine may divert attention and concerns of health care system from efforts to remedy the foundational causes of ill health such as poverty, obesity and education.

o) Health care costs: Genetic mapping of a population, and analyses of data, securing the information and deriving treatment recommendation are very costly which can be readily hampered by budgetary constraints of high dynamic economies. Further, the costs of converting the intellectual capital to therapeutic modalities must also be taken into account and built into the health care system. 
p) Finally, it is noteworthy that the concept of precision medicine may be a repackaging of the ideals advanced by the human genome project in 2000. The hope was to identify genetic markers for the ultimate objective of developing novel biomarkers and overcome perceived therapeutic deficiencies and overcome the pressing issue of non-responders. However, this noble objective has not been fully materialised yet, leading one to the question: Is precision medicine "old vine in a new bottle"?

In conclusion, precision medicine could potentially improve preventive methods and therapeutic options. However, a number of challenges remain as alluded to above-see also the cartoon in Fig. 1. Precision medicine will have to demonstrate consistency, coherence, comprehensiveness, clarity and relevance to every individual and community impacted by these developments in medical research and treatment. Will precision medicine deliver all that it promises? With increasing shrinking of financial resources, is it wise to invest millions of dollars/ euros in an approach for which its risks versus benefits ratio does not ring a satisfactory bell? Perhaps, stakeholders and authorities would be better off to further invest and focus on the already established concepts of "predictive, preventive and personalised medicine."

\section{Predictive, preventive and personalised medicine (PPPM)}

The paradigm shift from "unPPPM" to "PPPM"

The above described great plurality of approaches indicates a broad understanding of clear deficits which do exist in currently applied medical services and attempts of diverse professional groups to remedy the deficits. On the other hand, there is an increasing level of understanding that persisting deficits carry a fundamental character and, therefore, cannot be solved by superficial modifications of health care systems facilitating individual technologies such as "cancer genomics" by "precision medicine."

Global deficits are well-defined and described elsewhere as unpredictable, unpreventable and impersonal medicine [20, 21]. It is evident that a paradigm shift is needed to move from "reactive" to "predictive, preventive and personalised medicine" as a new philosophy

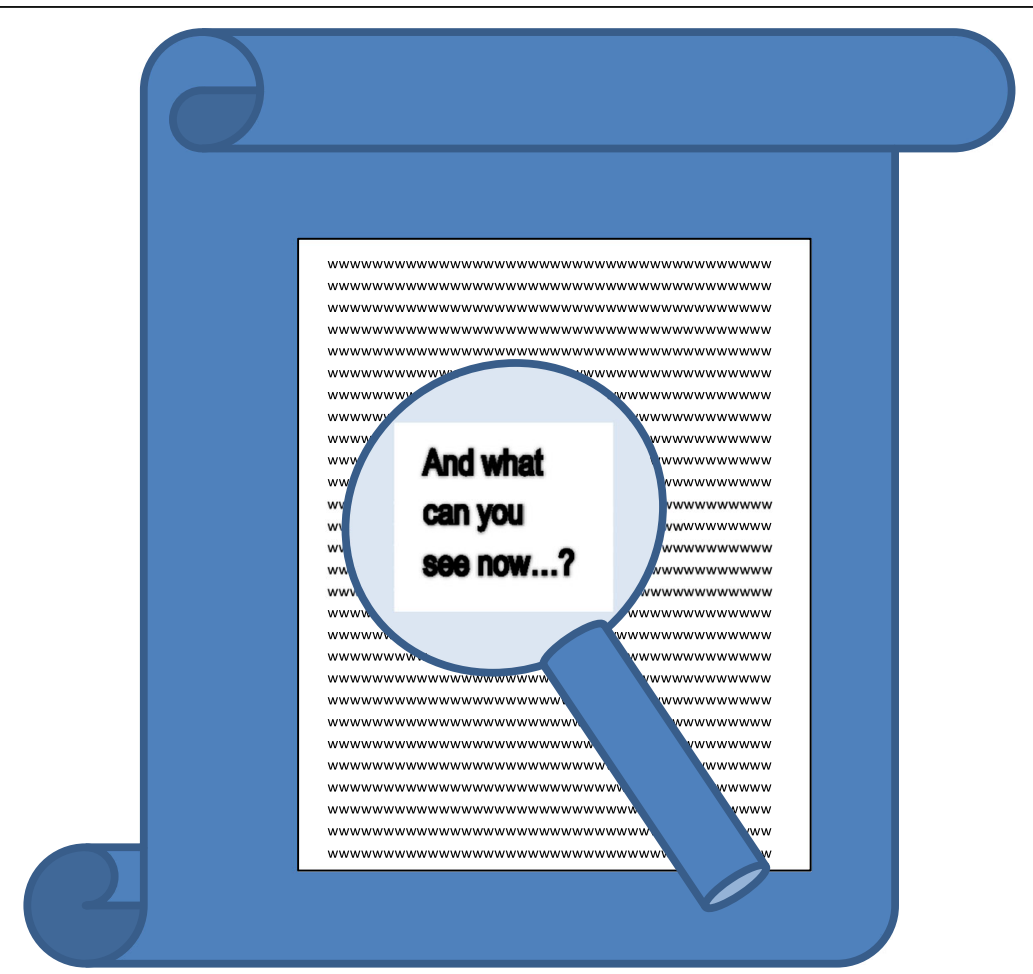

Fig. 1 "Precision" itself does not guarantee for better understanding of an issue. An old wise dictum adopted by all European cultures/languages warns - one cannot see the forest for the trees. Hence, technologically driven higher resolution of individual elements does not automatically mean that you can better recognise the complex problem which you are looking for, particularly when the zoomed element (the tree) is prioritised and/or pulled out from the overall context or zooming itself makes the complete picture (the forest/multifactorial issue) unreadable. Contextually, better understanding of the complexity in medicine is not guaranteed by "precision medicine" itself. Advanced health care demands a close cooperation between all issue-related fields, integration of multidisciplinary knowledge and innovative technologies based on long-term strategies and concepts considering interests of patients, professionals and society at large 
covering both "health care" and "disease care", promoting an integrated approach combining advantages of individual bio/medical fields and technologies and consolidating a multi-professional collaboration.

New paradigm has been created by the EPMA experts as published earlier [22] - see Fig. 2.

\section{Particular emphasis on ethics in PPPM}

Sometimes, we, living in the XXI century, forget what was taught when the first universities were established at the birth of XI century: "Never discuss about names," "Never enter an onomatomachia", that is, never enter a

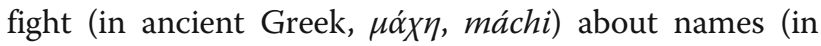
ancient Greek, óvou $\alpha$, ónoma). Sometimes, the meanings of the names "person-centred medicine," "individualised medicine," "stratified medicine," "precision medicine," "personalised medicine," etc. are not so sharp; sometimes, they intersect, but it is not clear until which point.

This is not a void and abstract "philosophical" question (actually, over the centuries and the millennia, the philosophers never thought that a question of names was a genuine philosophical question). This is a matter of money, a matter of grants, and a matter of power. This means that the onomatomachia now occurring in the field of contemporary biomedicine is actually a war for money and for power. But the citizens, in particular a subset of them, that is, the diseased citizens, are totally disinterested about it, even if, unfortunately, negative side effects of this war affect them. Citizens are interested in a personalised care, whatever this could precisely mean [23].
This means that beside a scientifically well-founded medical approach facing their unique potential or actual disease, they wish that their unique biography could be taken into account as well. Yet let us put aside for a while the biographical part (i.e. the age, gender, cultural, ethnic, religious, socio-economic diversity) of an actual or potential patient, even if we know, from epigenomics, that patients' lifestyles and the environments in which they live are extremely impacting their quality of life and their actual or potential diseases. Let us focus on the "medical" part.

A citizen with an actual or potential disease wants a medicine in which he/she is at the centre, a medicine which is tailored on his/her polymorphism, a medicine which is able to provide him/her with the right therapy, in the right dose, at the right moment, for the right period of time. But he/she also wants a medicine which is able to predict and prevent possible diseases. $\mathrm{He} / \mathrm{she}$ is not interested in the way in which this kind of medicine is called. But he/she is interested in understanding why it is called in that way, in order to appreciate its potential ability to restore health. And this is the real advantage of speaking in terms of predictive, preventive and personalised medicine: the actual or potential patient understands what is going on!

Nevertheless, there is something more. The PPPM lends itself to an over-arching umbrella under which the main ethical issues of contemporary biomedicine could be positively tackled. Certainly, a predictive and preventive approach could imply several ethical problems linked, for example, to overdiagnosis and overtreatment, detection of incidental findings, psychological burden

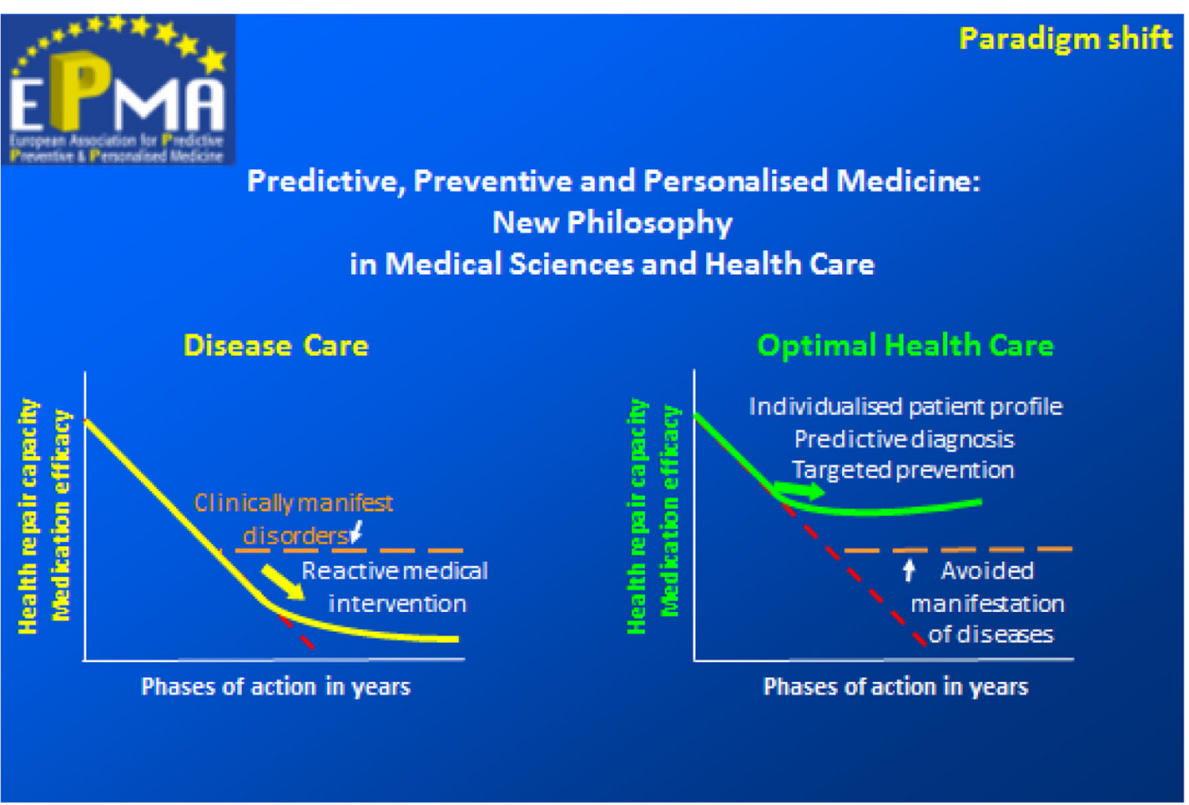

Fig. 2 Paradigm shift from "reactive" to "predictive, preventive and personalised medicine" 
and severe existential choices connected with the knowledge of the probability of a possible disease affecting us or our offspring and lineages, or connected with our reproductive choices, etc. Contextually, PPPM plays a crucial role as the optimal medical partner of a serious ethical counselling (and here, the patient's biography plays its main role) offered to actual or potential individual patients, in order to empower them to make an infirmed choice about the diagnostic, surveillance or therapeutic path to take, especially whenever these paths intersect ethical or existential problematic situations that they have to solve [24].

\section{Towards scientific excellence and practical PPPM} implementation: special professional focuses by the EPMA This subsection is based on the well-elaborated PPPM aspects evidently advancing medical sciences and health care. Corresponding professional statements have been approved by the association within the fundamental document resulting from the EPMA Summit 2014 under the auspices of the presidency of Italy in the EU [6].

\section{PPPM in cancer: the key questions puzzling medical sciences and advancing health care}

The majority of people may carry hardly detectable micro- and asymptomatic tumour lesions as it has been demonstrated by a series of detailed autopsy studies. However, those lesions do not necessarily progress into clinically manifest oncologic diseases. Furthermore, there is a phenomenon of the so-called metastatic inefficiency, due to less than $1 \%$ of all disseminated and circulated tumour cells which have a potential to form secondary and distanced tumours (metastatic disease) [25]. Contextually, the key question puzzling modern predictive, preventive and personalised medicine in oncology is how to predict and effectively protect against clinical manifestation of the disease by distinguishing between "silent" carriers of tumour lesions and patients who are predisposed to a disease development and progression. The clue might be a "fertile" microenvironment that effectively supports the tumorigenesis, tumour invasiveness and aggressive metastatic disease [26]. The mechanisms "fertilising" the microenvironment for the cancer advancement are well-addressed by innovative PPPM strategies in cancer [26-29].

\section{PPPM advancements in CVD management: a global health issue}

Currently, the CVD-related health burden is the most severe in developed countries and becomes overgrown in developing countries as well. The main reason for that is that the chronic disease stages, multifactorial diseases and comorbidities are not adequately addressed, since they do not follow the PPPM principles in currently practised health care systems [30]. An advanced CVD management is needed at both population and individual levels considering complex cardiovascular risk factors, co-morbidities, individualised patient profiles, optimised screening programmes and innovative preventive strategies. Chronic suboptimal health conditions such as primary vascular dysregulation (Flammer syndrome) may be relevant for a number of predispositions and severe pathologies with poor outcomes [31-33]. Consequently, the promotion of PPPM in CVD management is a global health issue [34-36].

\section{Global epidemic of diabetes type 2-twenty-first century disaster and PPPM solutions}

Current epidemiologic studies report about over 400 million of diabetes mellitus (DM) diseased patients worldwide. A big portion of DM cases remains undiagnosed. More and more teenagers are affected by DM type 2 . The global epidemic of DM type 2 places an alarming burden on health care systems. The consequent challenges and costs overload both developed and developing countries and economies. An effective implementation of PPPM concepts to diabetes care is due long ago. EPMA emphasises the need to address the all-encompassing complex approach for population screening, primary, secondary and tertiary care benefiting non-diseased individuals, predisposed subgroups and affected patient cohorts including those with comorbid pathologies such as CVD, cancer, and neurological, neuropsychiatric and neurodegenerative diseases (NNND) amongst others [6, 35, 37, 38].

\section{PPPM advancing the comprehensive area of NNND}

In a very few years, NNND are predicted to represent the majority of socially and economically devastating disorders and diseases. Multifactorial physical and cognitive disability of NNND-affected patient cohorts results from individual interplay of genetic, epigenetic and environmental risk factors. Contextually, the comprehensive area of NNND demands new strategies which would create a robust platform for the cost-effective medicine of future NNND management [39-42]. Consequently, the advanced PPPM concepts do place particular emphasis on primary prevention by the identification of predisposed individuals, improved patient stratification and treatments tailored to the person [6]. However, new regulations and innovative reimbursement programmes are mandatory to prompt an effective implementation of the above listed concepts.

\section{Rare disease (RD) management: proof-of-principles for personalised medical care}

Although an entire spectrum of RDs affects many millions of people worldwide (e.g. in Europe, there are at 
least 30 million patients), currently, no appropriate diagnostic and treatment approaches are available for most of afflicted with individual RDs. The majority of RDs can be diagnosed in prenatal and early postnatal periods. Due to the genetic background of most RD pathologies, the multimodal diagnostic and treatment approaches propagated by PPPM are instrumental for personalisation of RD management [43].

\section{Ancient medical traditions "reinforced" by innovative PPPM concepts}

PPPM creates a unique platform for "reinforcing" traditional approaches of the ancient medicines (TCAM). PPPM-TCAM hybrid demonstrates a great potential in person-centred and participatory medicine, disease prediction in individuals with suboptimal health condition, targeted prevention and individualised treatments. If properly designed, PPPM-TCAM approach may be of particular value for health care systems that empowers communities and individuals $[3,44]$.

\section{Application of PPPM to the pain management benefiting all medical fields}

Pain management is the central issue for a variety of syndromes, acute, chronic and systemic disorders. Pain diagnostics and treatment are highly individual involved in a wide spectrum of suboptimal health conditions, early and advanced stages of developing pathologies and collateral diseases such as CVD, NNND, diabetes, and cancer. Application of PPPM concepts to advanced pain management demands multidisciplinary expertise considered in the context of improved health care economy and policy and direct benefits to the patient [45-47].

\section{Impacts of the oral and dental health: novelty by PPPM concepts}

On the one hand, dental diseases are frequently caused by systemic disorders such as diabetes mellitus. On the other hand, dental and oral pathologies are both early indicators and risk factors for a variety of multifactorial diseases. This includes pre-term birth, a spectrum of vascular pathologies, stroke, heart and lung disease, diabetes mellitus with comorbidities, some types of cancer, neurological disorders and several mental disorders such as depression, anxiety, anorexia and even bulimia. Therefore, investigation of the cause-and-effect relationships between oral and dental diseases on the one hand and multifactorial systemic disorders on the other hand is a prerequisite for predictive, preventive and personalised medicine in the multidisciplinary fields of dental and oral health care [48-52].

\section{Environmental factors in a sensitive balance between health} and disease

There is a highly sensitive interplay between a genetic component, epigenetic regulations and environmental factors that determines a sensitive balance between health and disease in individuals. Unfortunately, environment is still a largely neglected topic in health care. PPPM approach aims to develop an appropriate knowledge and technological skills for promoting affordable strategies in the emerging fields of environmental risk factors, epidemiology, healthy lifestyle, individualised nutrition, food technology and culture in a framework of cost-effective health care [29, 47, 53-55].

\section{Robust PPPM platform to advance regenerative medicine}

Prediction and personalisation in regenerative medicine are prerequisites for improved individual outcomes. Hence, in order to optimally match the donor to recipient and assess individual risks, a successful transplantation requires valid pathology-specific pre- and post-transplantation biomarker panels tailored to the individual. Long waiting lists of patients worldwide reflect major problems and current deficits, which require PPPM-related solutions advancing this medical area on the global scale [6]. Individual components of the overall management leading to substantially increased allograft survival and decreased patient morbidity are an improved donor-recipient matching, individual risk assessment for chronic allograft damage, prediction of graft accommodation and creation of personalised immunosuppressive algorithms.

\section{Body culture and sports medicine (BCSP) effectively promoted by PPPM}

PPPM strategies in BCSP are based on optimisation of the relationship between individual genetic predispositions and modifiable risk factors (nutrients, physical activity, lifestyle, etc.). Therefore, the main tools are individualised physical exercises and therapy algorithms, healthy balance between body tension and relaxation, optimised sleep algorithms according to individual circadian rhythm, innovative rehabilitation approaches, amongst others. Antidoping control and effective measures are mandatory for PPPM implementation in advanced BCSP. High-quality research based on measurable effects utilising multilevel biomarker panels is effectively promoted by PPPM in BCSP with a particular focus on individually tailored interventions [56-59].

\section{Translational medicine: a powerful bridge between PPPM science and implementation}

There are many scientific fields which, on a daily basis, provide a great knowledge potentially useful for advanced medical services. However, a number of scientific articles and valuable patents remain unused. The "bottleneck" 
between the sciences and application has many reasons including economic circumstances and missing political regulations. In order to effectively promote the translational medicine as the "catalyser" for practical implementation of the accumulated scientific achievements, EPMA creates a robust platform for an effective dialogue between PPPM relevant professional groups on the one side, and industry and policy-makers on the other side-for more information, see the main documents of the association $[6,35]$. The main goal is to translate knowledge from studies at the bench side to care at the bedside by following mechanism: from discovery to health application, to evidence-based guidelines, to advanced health care services and finally to health impacts for the patient $[60,61]$.

\section{Information and communication technologies (ICT) resulting in cost-effective modernisation of health care}

A holistic presentation of individuals and discoursed health condition by ICT approach implies a redesign of health care services. The ICT support is the prerequisite for an effective PPPM by disease modelling, individualised patient profiles, optimised diagnostic and treatment approaches. The ICT tools include mathematical modelling methods, such as probabilistic relational models and process models, prediction of a disease development, precise patient stratification, creation of the multimodal diagnostic approaches, elaboration of the best possible therapy algorithms, an estimation of individual outcomes, distanced patient monitoring, advanced avatar technologies, and bid data management amongst others. Contextually, ICT is anticipated to result in profound and cost-effective modernisation of health care benefiting the patient, health care providers and society at large $[28,29,62-66]$.

\section{The crucial role of multilevel diagnostics in PPPM}

Accumulating evidence demonstrates that an ideal biomarker does not exist. The role of multilevel diagnostics is to provide maximum clinically relevant information by utilising pathology- and stage-specific biomarker panels at the level of medical imaging, subcellular imaging, multi-omics and relevant hybrid technologies. Integrating this information allows for targeted prevention and personalised treatment regimes, avoiding unnecessary drug toxicity, decreasing negative side-effects and reducing morbidity [27-29, 62, 67-69].

\section{Laboratory medicine in PPPM concepts: from passive assistance to active advising}

Delayed intervention, untargeted medication, overdosed patients and ineffective treatments, amongst others, are the deficits in currently pursued medical services that demand a revised role of laboratory medicine in health care systems. The laboratory services should become more complex, advancing multifactorial analysis. Such a complex analytics should result in recommendations and active advising for clinicians in order to more accurately interpret health-related data of the individual/patient. Therefore, an effective ICT support (see the "Information and communication technologies (ICT) resulting in cost-effective modernisation of health care" section) is mandatory. Practical implementation of novel and complex laboratory tests certainly should be considered from the viewpoint of their reasonability, cost-effectiveness and value added to a data interpretation. Smart laboratory investigation strategies and all-encompassing data interpretation are essential for an appropriate relationship between laboratory medicine and clinicians acting handin-hand as the decision makers responsible for better individual outcomes [70-75].

\section{Well-regulated biobanking and biopreservation is pivotal for future progress in PPPM}

For the future progress in development of novel biomarker panels, predictive and prognostic technologies and personalisation of treatment regimes, an internationally valid biobanking and biopreservation are essential. A proper creation of that is currently an ongoing process in PPPM [66]. Considering individual types of biological material (tissue, saliva, blood and cell samples, DNA, RNA, proteins, metabolites, etc.), the major challenges are due to:

- Consideration of ethical aspects including privacy- and security-related issues [76, 77]

- Adequate national and international regulations

- Optimised protocols for collecting, storing and retrieving the samples

- High analytical quality of all the process of biobanking and biopreservation

- Adequately organised clinical/patient databases.

An effective support by the advanced ICT systems for smoothly run processing and adequate data interpretation is crucial for the clinical utility of biobanks [66].

\section{Design of professional interactome in PPPM}

PPPM carries highly multi- and interdisciplinary character and demonstrates high level of international cooperation. Consequently, related networking demands an effective interaction amongst professional groups as well as between health care professionals and patient groups and policy-makers. All these groups currently do "speak different languages," which may create some communication barriers, however, reinforcing each group's perspective to reach higher level of understanding and cooperation in PPPM framework. The specific output of this design activity is the so-called professional interactome [78]. The 
PPPM-related interactome represents the most optimal model of health care organisation with significantly increased quality of multilevel communication and cooperation resulting in improved individual patient outcomes and health care economy (see the "Advanced business models for PPPM concepts in health care" section).
Education as the heart of the PPPM-related scientific excellence and successful practical implementation

The ultimate goal is to create a new culture in the health care sector and to promote high level of professionalism by new generations of healthcare-givers who will be capable to implement an all-encompassing approach to

Table 1 Conclusions and expert recommendations

\begin{tabular}{lll}
\hline Term & Advantages & Limitations \\
\hline Traditional, & Increases the own repair capacity & Less effective for disease care; in \\
complementary & of the human body; deals with & manifest pathologies can be applied \\
and alternative & natural products and physiological & to complement conventional \\
medicine, TCAM & approaches; is dedicated to disease & treatments such as surgery, \\
& $\begin{array}{l}\text { prevention and well-being; highly } \\
\text { effective at the level of suboptimal }\end{array}$ & $\begin{array}{l}\text { chemo-therapy, etc.; cultural barriers } \\
\text { can exist when TCAM is introduced }\end{array}$ \\
& by the country of origin to other \\
& countries with sufficiently different \\
& cultural habits
\end{tabular}

Person-centred medicine, PCM

Individualised medicine, IM

Stratified medicine, SM

Personalised medicine, PM

Precision medicine PrecMed

Predictive preventive and personalised medicine, PPPM
Promotion of health as a state of physical, mental, social and spiritual well-being; potential for disease reduction; emphasis on science and humanism; PCM promotes approaches to health improvement, respect and responsibility at individual and community levels

IM propagates a holistic approach by acknowledging multidimensional interaction between internal and external risk factors which vary from individual to individual.

More targeted treatments according to individual patient subtype; stratified treatment algorithms

Actually considered best possible medical treatments adapted to the needs of the patient

PrecMed attracts attention of policy-makers to problems persisting in medical services; additionally released budgets in medical sciences; increased publicity for disease care; potentially increased cooperation level between individual medical fields

PPPM is a really complex all-encompassing approach combining advantages of the above listed individual approaches and minimising their specific disadvantages; clear concepts demonstrating the highest level of maturity; the most optimal strategies considering interests of healthy individuals, subpopulations, patient cohorts, health care systems and society as a whole.

Optimal application and unique niche

$>$ Diagnosis and treatment of suboptimal health conditions

$>$ Cost-effective preventive medicine

$\triangle$ Emphasises well-being

$>$ Pain management

$>$ Complementary treatments

$>$ Cultural traditions of the country of origin

Realisation of the ideals promoted by PCM demands clear definitions and validated strategies

$>$ Health care philosophy

$>$ Mental maturation of society at large

$>$ Integration of sciences and humanism

$>$ Promotion of health

$>$ Promotion of respect and responsibility in the society

IM is clearly focused on individualisation of standardised intervention, but it provides less developed concepts of predictive and preventive medicine, if any.

Although being an extremely important instrument, SM represents just one step towards "personalised medicine."

Concepts of PM are adapted to "disease care" but not to "health care."

Politically motivated initiative utilising advantages of already existing and above listed approaches; strong limitations by selectively promoted technological focuses (e.g. genomics); unclear integration strategies in medicine; unclear cost-effectiveness, benefits to individual patient cohorts and overall health care economy

PPPPM is considered as the "medicine of the future" which needs the paradigm change for entire spectrum of medical research and services, improved professional and general educational levels, new economic and application models for both disease and health care.

$\triangleright$ Cost-effective medical services and $\triangleright$ Holistic approach to standardised intervention

$\gg$ Patient categorisation and modelling

$\gg$ Cohort subgrouping

$\gg$ Patient stratification

Semi-personalised medicine compromising between standardisation and individualisation in medicine

$\gg$ Potentially improved clinical impacts of specific areas such as genomics

$\gg$ Potential technological integration in medical fields

$\gg$ Potentially improved outcomes in some patient cohorts

$\triangleright$ Desirable versus current health care systems

$\gg$ Predictive medicine

$\triangleright$ New spectrum of screening programmes

$\gg$ Targeted prevention

$\triangleright$ Currently unmet needs of healthy subpopulations and patient cohorts optimised health care economy

$\triangleright$ New dimension of professional interests

$\triangleright$ New scale of the knowledge integration

$\gg$ Highly motivated technological innovation

$\triangleright$ Highly motivated interdisciplinary and multidisciplinary cooperation

$>$ Individualised patient profiling

$\gg$ Active participation of patients in the health care process 
patient care recognising the complexity and individuality of the human being. In order to promote innovative educational programmes, the following worldwide pioneer initiatives have been developed:

- The EPMA Journal regularly updates information about medical innovations and advanced health care providing expert recommendations in predictive diagnostics, targeted preventive measures and individualised treatment algorithms (https://epmajournal.biomedcentral.com/ and http://www.springer.com/biomed/journal/13167).

- Advances in predictive, preventive and personalised medicine (http://www.springer.com/series/10051): this book series, launched in 2012, provides an overview of complex strategies, innovative technologies, novel biomarker panels, and multidisciplinary aspects of advanced biomedical approaches in individual PPPM areas and health care as a whole. New technologies and guidelines are provided for medical ethics, early and predictive diagnostics, targeted prevention, treatments tailored to the person, health care organisation and economy. This book series is intended to serve as a reference source for multidisciplinary research and the health care industry with special emphasis on advanced health promotion and cost-effective treatment of diseases.

\section{Advanced business models for PPPM concepts in health care} If left unchanged, a long-term poor cost-effectiveness may lead to economic collapse of current health care systems with persisting archaic business models. Across Europe, there is a great diversity of systems, payment models and reimbursement schemes in health care [72]. This imposes a highly fragmented market. On the one hand, there is a need for policy dialogue in order to achieve improved structure and delivery. On the other hand, advanced business models are required, in order to motivate

- Healthcare-givers to apply more individualised diagnostic and treatment approaches

- Healthy individuals and patients to accept greater responsibility towards their own health condition

- Industry to create novel products for health support, promotion and monitoring

- Policy-makers for smart long-term regulations in health care sector such as an effective promotion of increased health literacy in population, advanced screening programmes and new reimbursement models for individual subpopulations and professional groups
- Finally, the society at large to reinvest budgets focused on the most cost-effective health promotion and primary health care.

In view of economic strain and the ageing populations, PPPM-related innovation in health care systems is critical for keeping the high quality of health care affordable and sustainable on European and global scale. Since its very beginning, EPMA is systematically working on the economy of PPPM that is pivotal for advancing health care on European and global scale $[6,8,20-22,28,30$, $35,37,40,71,72,79-82]$.

\section{Conclusions and expert recommendations}

Concluding remarks are summarised in Table 1 in form of advantages and limitations listed for individual types of medicines analysed in this paper followed by recommendations for their most optimal application.

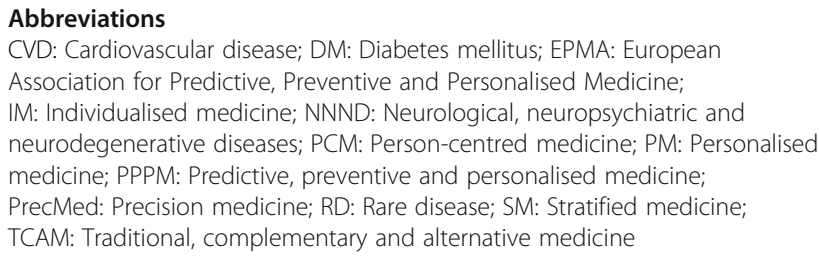

\section{Acknowledgements}

The position paper is created on behalf of the European Association for Predictive, Preventive and Personalised Medicine, EPMA, Brussels. The nominated working groups (authors of the position paper) thank all EPMA members for their excellent professional expertise and a series of PPPM-related articles to which current paper refers.

\section{Funding}

No funding has been provided.

\section{Availability of data and materials}

Data sharing not applicable to this article as no datasets were generated or analysed during the current study.

\section{Authors' contributions}

All authors have performed literature search and equally contributed to the concepts presented in the paper. OG has drafted the paper. $B B, G B, W W, R B, M K, K K, M M$ and $V C$ have contributed to the contents of individual subsections. All authors read and approved the final manuscript.

\section{Competing interests}

The authors declare that they have no competing interests.

\section{Consent for publication}

Not applicable.

\section{Ethics approval and consent to participate}

Not applicable.

\section{Author details}

${ }^{1}$ European Association for Predictive, Preventive and Personalised Medicine, Brussels, Belgium. ${ }^{2}$ Radiologic Department, Rheinische Friedrich-Wilhelms-University of Bonn, Sigmund-Freud-Str. 25, 53105 Bonn, Germany. ${ }^{3}$ Breast Cancer Research Centre, Rheinische Friedrich-Wilhelms-University of Bonn, Bonn, Germany. ${ }^{4}$ Augusta University, Augusta, GA, USA. ${ }^{5}$ Department of Surgery, School of Medicine, Augusta University, Augusta, GA, USA. 'Dipartimento di 
Scienze Biomediche e Chirurgico Specialistiche, Università di Ferrara, Via Fossato di Mortara, 64A, 44121 Ferrara, Italy. ${ }^{7}$ Institute for Advanced Study, Technische Universität München, Garching bei München, Germany. ${ }^{8}$ School of Medical Sciences, Edith Cowan University, Perth, Australia. 'Beijing Municipal Key Laboratory of Clinical Epidemiology, Capital Medical University, Beijing, China. ${ }^{10}$ WHO Expert Panel (Member), Geneva, Switzerland. " ${ }^{11}$ Global Health Epidemiology Reference Group (GHERG), Edinburgh, UK. ${ }^{12}$ Clinical hospital "Pheophania" of State Affairs Department, Kyiv, Ukraine. ${ }^{13}$ Zabolotny Institute of Microbiology and Virology, National Academy of Sciences of Ukraine, Kyiv, Ukraine.

${ }^{14}$ European Medical Association, Brussels, Belgium.

Received: 24 September 2016 Accepted: 11 October 2016 Published online: 25 October 2016

\section{References}

1. Xue CC. Traditional, complementary and alternative medicine: policy and public health perspectives. Bull World Health Organ. 2008;86(1):77-8. doi:10. 2471/BLT.07.046458.

2. Fokunang CN, Ndikum V, Tabi OY, Jiofack RB, Ngameni B, Guedje NM, Tembe-Fokunang EA, Tomkins P, Barkwan S, Kechia F, Asongalem E, Ngoupayou J, Torimiro NJ, Gonsu KH, Sielinou V, Ngadjui BT, Angwafor 3rd F, Nkongmeneck A, Abena OM, Ngogang J, Asonganyi T, Colizzi V, Lohoue J, Kamsu-Kom. Traditional medicine: past, present and future research and development prospects and integration in the National Health System of Cameroon. Afr J Tradit Complement Altern Med. 2011;8(3):284-95.

3. Wang W, Russell A, Yan Y, Global Health Epidemiology Reference Group (GHERG). Traditional Chinese medicine and new concepts of predictive, preventive and personalized medicine in diagnosis and treatment of suboptimal health. EPMA J. 2014;5(1):4. doi:10.1186/1878-5085-5-4.

4. Lang CL, Wang MH, Hung KY, Chiang CK, Lu KC. Altered molecular repertoire of immune system by renal dysfunction in the elderly: is prediction and targeted prevention in the horizon? EPMA J. 2013:4(1):17 doi:10.1186/1878-5085-4-17.

5. Kupaev V, Borisov O, Marutina E, Yan YX, Wang W. Integration of suboptimal health status and endothelial dysfunction as a new aspect for risk evaluation of cardiovascular disease. EPMA J. 2016;7(1):19. doi:10.1186/ s13167-016-0068-0.

6. Golubnitschaja O, Costigliola V, EPMA. EPMA summit 2014 under the auspices of the presidency of Italy in the EU: professional statements. EPMA J. 2015;6(1):4. doi:10.1186/s13167-015-0026-2.

7. Roberti di Sarsina P, Tassinari M. Person-centred healthcare and medicine paradigm: it's time to clarify. EPMA J. 2015;6(1):11. doi:10.1186/s13167-015-0033-3.

8. Fischer T, Langanke M, Marschall $P$, Michl S, editors. Individualized medicine, advances in predictive, preventive and personalised medicine, vol. 7. Springer Dordrecht Heidelberg New York London; 2015. ISBN 978-3-319-11718-8.

9. Realising the potential of stratified medicine. The academy of medical sciences 2013, https://www.acmedsci.ac.uk/viewFile/51e915f9f09fb.pdf (viewed on September 16th 2016)

10. Marino N, Woditschka S, Reed LT, Nakayama J, Mayer M, Wetzel M, Steeg PS. Breast cancer metastasis issue for the personalization of its prevention and treatment. Am J Path. 2013;183(4):1084-95. doi:10.1016/j.ajpath.

11. Jameson JL, Longo DL. Precision medicine-personalized, problematic, and promising. N Engl J Med. 2015;372(23):2229-34. doi:10.1056/ NEJMsb1503104.

12. Collins FS, Varmus H. A new initiative on precision medicine. N Engl J Med. 2015;372(9):793-5. doi:10.1056/NEJMp1500523.

13. Rubin R. Precision Medicine: The future or simply politics? JAMA. 313(11): 1089-91. doi:10.1001/jama.2015.0957.

14. Patel K. Precision medicine: pros \& cons. February 1st 2015. https://kirtipatel.com/ 2015/02/01/precision-medicine-blessing-or-curse/. Accessed 21 Sept 2016.

15. What are some of the challenges facing precision medicine and the precision medicine initiative? https://ghr.n/m.nih.gov/primer/ precisionmedicine/challenges. Accessed 21 Sept 2016.

16. Cardon LR, Harris T. Precision medicine, genomics abd drug discovery. Hum Mol Genet. 2016. doi:10.1093/hmg/ddw246.

17. Aerts HJWL. The potential of radiomic-based phenotyping in precision medicine: a review. JAMA Oncol. 2016. doi:10.1001/jamaoncol.2016.2631.

18. Nazha A, Sekeres MA. Precision medicine in myelodysplastic syndromes and leukemias: lessons from sequential mutations. Annu Rev Med. 2016; doi:10.1146/annurev-med-062915-095637.
19. Precision medicine initiative and data security. May 25th 2016. https://www. whitehouse.gov/blog/2016/05/25/precision-medicine-initiative-and-datasecurity. Accessed 21 Sept 2016.

20. Andrews RJ, editor. Too big to succeed-profiteering in American medicine. USA: iUniverse; 2013. ISBN: 978-1-14759-7130-9.

21. Andrews RJ, Quintana LM. Unpredictable, unpreventable and impersonal medicine: global disaster response in the 21st century. EPMA J. 2015;6(1):2. doi:10.1186/s13167-014-0024-9.

22. Golubnitschaja $\mathrm{O}$, editor. Predictive diagnostics and personalized treatment: dream or reality. New York: Nova; 2009. ISBN 978-1-60692-737-3.

23. Cornetta K, Brown CB. Perspective: balancing personalized medicine and personalized care. Acad Med. 2013;88(3):309-13. doi:10.1097/ACM. Ob013e3182806345

24. Boniolo G, Sanchini V, editors. Ethical counselling and medical decision-making in the era of personalised medicine. A practice-oriented guide. Springer Dordrecht Heidelberg New York London; 2016. ISBN 978-3-319-27690-8.

25. Redig AJ, McAllister SS. Breast cancer as a systemic disease: a view of metastasis. J Intern Med. 2013;274(2):113-26. doi:10.1111/joim.12084.

26. Cox TR, Rumney RMH, Schoof EM, Perryman L, Høye AM, Agrawal A, Bird D, Latif NA, Forrest H, Evans HR, Huggins ID, Lang G, Linding R, Gartland A, Erler JT. The hypoxic cancer secretome induces pre-metastatic bone lesions through lysyl oxidase. Nature. 2015;522(7554):106-10. doi:10.1038/nature14492.

27. Golubnitschaja O and Sridhar KC. Liver metastatic disease: new concepts and biomarker panels to improve individual outcomes. Clin Exp Metastasis. 2016; doi:10.1007/s10585-016-9816-8

28. Grech G, Zhan X, Yoo BC, Bubnov R, Hagan S, Danesi R, Vittadini G, Desiderio DM. EPMA position paper in cancer: current overview and future perspectives. EPMA J. 2015;6(1):9. doi:10.1186/s13167-015-0030-6.

29. Golubnitschaja O, Debald M, Yeghiazaryan K, Kuhn W, Pešta M, Costigliola V, Grech G. Breast cancer epidemic in the early 21st century: evaluation of risk factors, cumulative questionnaires and recommendations for preventive measures. Tumor Biol. 2016; doi:10.1007/s13277-016-5168-x.

30. Brunner-La Rocca HP, Fleischhacker L, Golubnitschaja O, Heemskerk F, Helms T, Hoedemakers T, Allianses SH, Jaarsma T, Kinkorova J, Ramaekers J, Ruff P, Schnur I, Vanoli E, Verdu J, Zippel-Schultz B. Challenges in personalised management of chronic diseases-heart failure as prominent example to advance the care process. EPMA J. 2016;7:2. doi:10.1186/s13167-016-0051-9.

31. Konieczka K, Ritch R, Traverso CE, Kim DM, Kook MS, Gallino A, Golubnitschaja O, Erb C, Reitsamer HA, Kida T, Kurysheva N, Yao K. Flammer syndrome. EPMA J. 2014;5(1):11. doi:10.1186/1878-5085-5-11.

32. Golubnitschaja O, Debald M, Kuhn W, Yeghiazaryan K, Bubnov RV, Goncharenko VM, Lushchyk U, Grech G, Konieczka K. Flammer syndrome and potential formation of pre-metastatic niches: a multi-centred study on phenotyping, patient stratification, prediction and potential prevention of aggressive breast cancer and metastatic disease. EPMA J. 2016;7 Suppl 1:A25.

33. Konieczka K, Koch S, Binggeli T, Schoetzau A, Kesselring J. Multiple sclerosis and primary vascular dysregulation (Flammer syndrome). EPMA J. 2016;7:13. doi:10.1186/s13167-016-0062-6

34. Iso H. Promoting predictive, preventive and personalized medicine in treatment of cardiovascular diseases. EPMA J. 2011;2(1):1-4. doi:10.1007/s13167-011-0075-0.

35. Golubnitschaja O, Costigliola V, EPMA. General report \& recommendations in predictive, preventive and personalised medicine 2012: white paper of the European association for predictive, preventive and personalised medicine. EPMA J. 2012;3(1):14. doi:10.1186/1878-5085-3-14.

36. Helms TM, Duong G, Zippel-Schultz B, Tilz RR, Kuck K-H, Karle CA. Prediction and personalised treatment of atrial fibrillation-stroke prevention: consolidated position paper of CVD professionals. EPMA J. 2014;5(1):15. doi:10.1186/1878-5085-5-15.

37. Mozaffari MS, editor. New strategies to advance pre/diabetes care: integrative approach by PPPM, advances in predictive, preventive and personalised medicine, vol. 2. Springer Dordrecht Heidelberg New York London; 2013. ISBN 978-94-007-5970-1.

38. Golubnitschaja O. Time for new guidelines in advanced diabetes care: paradigm change from delayed interventional approach to predictive, preventive and personalized medicine. EPMA J. 2010;1(1):3-12. doi:10.1007/s13167-010-0014-5.

39. Golubnitschaja O, Yeghiazaryan K, Cebioglu M, Morelli M, Herrera-Marschitz M. Birth asphyxia as the major complication in 
newborns: moving towards improved individual outcomes by prediction, targeted prevention and tailored medical care. EPMA J. 2011;2(2):197-210. doi:10.1007/s13167-011-0087-9.

40. Mandel S, editor. Neurodegenerative diseases: integrative PPPM approach as the medicine of the future, advances in predictive, preventive and personalised medicine, vol. 3. Springer Dordrecht Heidelberg New York London; 2013. ISBN 978-94-007-5865-0.

41. Sinnecker T, Kuchling J, Dusek P, Dörr J, Niendorf T, Paul F, Wuerfel J. Ultrahigh field MRI in clinical neuroimmunology: a potential contribution to improved diagnostics and personalised disease management. EPMA J. 2015;6(1):16. doi:10.1186/s13167-015-0038-y.

42. Polivka J, Polivka Jr J, Krakorova K, Peterka M, Topolcan O. Current status of biomarker research in neurology. EPMA J. 2016;7:14. doi:10.1186/s13167-016-0063-5

43. Özgüç M, editor. Rare diseases_-integrative PPPM approach as the medicine of the future, advances in predictive, preventive and personalised medicine, vol. 6. Springer Dordrecht Heidelberg New York London; 2015. ISBN 978-94-017-9213-4.

44. Roberti di Sarsina P, Alivia M, Guadagni P. Traditional, complementary and alternative medical systems and their contribution to personalisation, prediction and prevention in medicine-person-centred medicine. EPMA J. 2012;3(1):15. doi:10.1186/1878-5085-3-15.

45. Bubnov RV. Evidence-based pain management: is the concept of integrative medicine applicable? EPMA J. 2012;3(1):13. doi:10.1186/1878-5085-3-13.

46. Arai YC, Yasui H, Isai H, Kawai T, Nishihara M, Sato J, Ikemoto T, Inoue S, Ushida T. The review of innovative integration of Kampo medicine and Western medicine as personalized medicine at the first multidisciplinary pain center in Japan. EPMA J. 2014;5(1):10. doi:10.1186/1878-5085-5-10.

47. Moiseyenko W, Sukhorukov VI, Pyshnov GY, Mankovska IM, Rozova KV, Miroshnychenko OA, Kovalevska OE, Madjar SA, Bubnov RV, Gorbach AO, Danylenko KM, Moiseyenko Ol. Antarctica challenges the new horizons in predictive, preventive, personalized medicine: preliminary results and attractive hypotheses for multi-disciplinary prospective studies in the Ukrainian "Akademik Vernadsky" station. EPMA J. 2016;7:11. doi:10.1186/s13167-016-0060-8. Erratum in: EPMA J. 2016;7:17.

48. Cafiero C, Matarasso S. Predictive, preventive, personalised and participatory periodontology: "the 5Ps age" has already started. EPMA J. 2013;4(1):16. doi:10.1186/1878-5085-4-16.

49. Golubnitschaja O, Costigliola V. Dental health: EPMA recommendations for innovative strategies. EPMA J. 2014;5 Suppl 1:A119.

50. Kunin AA, Evdokimova AY, Moiseeva NS. Age-related differences of tooth enamel morphochemistry in health and dental caries. EPMA J. 2015;6(1):3. doi:10.1186/s13167-014-0025-8.

51. Tachalov W, Orekhova LY, Kudryavtseva TV, Isaeva ER, Loboda ES. Manifestations of personal characteristics in individual oral care. EPMA J. 2016;7:8. doi:10.1186/s13167-016-0058-2.

52. Qin X, Liu JY, Abdelsayed R, Shi X, Yu JC, Mozaffari MS, Baban B. The status of glucocorticoid-induced leucine zipper protein in the salivary glands in Sjögren's syndrome: predictive and prognostic potentials. EPMA J. 2016;7:3. doi:10.1186/s13167-016-0052-8.

53. Trovato GM. Behavior, nutrition and lifestyle in a comprehensive health and disease paradigm: skills and knowledge for a predictive, preventive and personalized medicine. EPMA J. 2012;3(1):8. doi:10.1007/s13167-012-0141-2.

54. Shapira N. Women's higher health risks in the obesogenic environment: a gender nutrition approach to metabolic dimorphism with predictive, preventive, and personalised medicine. EPMA J. 2013;4(1):1. doi:10.1186/1878-5085-4-1.

55. Richter K, Acker J, Adam S, Niklewski G. Prevention of fatigue and insomnia in shift workers-a review of non-pharmacological measures. EPMA J. 2016; 7:16. doi:10.1186/s13167-016-0064-4

56. Oja P, Titze S. Physical activity recommendations for public health: development and policy context. EPMA J. 2011;2:253-9.

57. Graf C. Preventing and treating obesity in pediatrics through physical activity. EPMA J. 2011;2(3):261-70. doi:10.1007/s13167-011-0091-0.

58. Schulte S, Rasmussen NN, McBeth JW, Richards PQ, Yochem E, Petron DJ, Strathmann FG. Utilization of the clinical laboratory for the implementation of concussion biomarkers in collegiate football and the necessity of personalized and predictive athlete specific reference intervals. EPMA J. 2016;7:1. doi:10.1186/s13167-016-0050-x

59. Trovato FM, Catalano D, Musumeci G, Trovato GM. 4Ps medicine of the fatty liver: the research model of predictive, preventive, personalized and participatory medicine-recommendations for facing obesity, fatty liver and fibrosis epidemics. EPMA J. 2014;5(1):21. doi:10.1186/1878-5085-5-21.

60. Younesi E, Hofmann-Apitius M. From integrative disease modeling to predictive, preventive, personalized and participatory (P4) medicine. EPMA J. 2013;4:23. doi:10.1186/1878-5085-4-23.

61. Drucker E, Krapfenbauer K. Pitfalls and limitations in translation from biomarker discovery to clinical utility in predictive and personalised medicine. EPMA J. 2013;4:7. doi:10.1186/1878-5085-4-7.

62. Hu R, Wang X, Zhan X. Multi-parameter systematic strategies for predictive, preventive and personalised medicine in cancer. EPMA J. 2013;4(1):2. doi:10.1186/1878-5085-4-2.

63. Lemke HU, Golubnitschaja O. Towards personal health care with model-guided medicine: long-term PPPM-related strategies and realisation opportunities within "Horizon 2020". EPMA J. 2014;5(1):8. doi:10.1186/1878-5085-5-8.

64. Berliner L, Lemke HU, VanSonnenberg E, Ashamalla H, Mattes MD, Dosik D, Hazin H, Shah S, Mohanty S, Verma S, Esposito G, Bargellini I, Battaglia V, Caramella D, Bartolozzi C, Morrison P. Model-guided therapy for hepatocellular carcinoma: a role for information technology in predictive, preventive and personalized medicine. EPMA J. 2014;5(1):16. doi:10.1186/1878-5085-5-16.

65. Berliner L, Lemke HU, editors. An information technology framework for predictive, preventive and personalised medicine, a use-case with hepatocellular carcinoma, advances in predictive, preventive and personalised medicine, vol. 8. Springer Dordrecht Heidelberg New York London; 2015. ISBN 978-3-319-12165-9.

66. Kinkorova J. Biobanks in the era of personalized medicine: objectives, challenges, and innovation: overview. EPMA J. 2016;7:4. doi:10.1186/s13167-016-0053-7.

67. Grech G, Grossman I, editors. Preventive and predictive genetics: towards personalised medicine, advances in predictive, preventive and personalised medicine, vol. 9. Springer Dordrecht Heidelberg New York London; 2015. ISBN 978-3-319-15343-8.

68. Hagan S, Martin E, Enríquez-de-Salamanca A. Tear fluid biomarkers in ocular and systemic disease: potential use for predictive, preventive and personalised medicine. EPMA J. 2016;7:15. doi:10.1186/s13167-016-0065-3.

69. Girotra S, Yeghiazaryan K, Golubnitschaja O. Potential biomarker panels in overall breast cancer management: advancements by multilevel diagnostics. Pers Med. 2016;13(5):469-84. doi:10.2217/pme-2016-0020.

70. Waerner $T$, Thurnher $D$, Krapfenbauer $K$. The role of laboratory medicine in healthcare: quality requirements of immunoassays, standardisation and data management in prospective medicine. EPMA J. 2010;1(4):619-26. doi:10.1007/s13167-010-0053-y.

71. Golubnitschaja O, Watson ID, Topic E, Sandberg S, Ferrari M, Costigliola V. Position paper of the EPMA and EFLM: a global vision of the consolidated promotion of an integrative medical approach to advance health care. EPMA J. 2013:4(1):12. doi:10.1186/1878-5085-4-12.

72. Costigliola V. Healthcare overview: new perspectives, advances in predictive, preventive and personalised medicine, vol. 1. Springer Dordrecht Heidelberg New York London; 2012. ISBN 978-94-007-4602-2.

73. Gahan PB. Circulating nucleic acids in early diagnosis, prognosis and treatment monitoring, advances in predictive, preventive and personalised medicine, vol. 5. Springer Dordrecht Heidelberg New York London; 2015. ISBN 978-94-017-9167-0.

74. Abraham J-A, Yeghiazaryan K, Golubnitschaja O. Selective internal radiation therapy in treatment of hepatocellular carcinoma: new concepts of personalization. Pers Med. 2016;13(4):347-60. doi:10.2217/pme-2016-0014.

75. Abraham J-A, Golubnitschaja O. Time for paradigm change in management of hepatocellular carcinoma: is a personalized approach on the horizon? Pers Med. 2016;13(5):455-67. doi:10.2217/pme-2016-0013.

76. Boniolo G, Di Fiore PP, Pece S. Trusted consent and research biobanks. Towards a "new alliance" between researchers and donors. Bioethics. 2012;26(2):93-100. doi:10.1111/j.1467-8519.2010.01823.x.

77. Sanchini V, Bonizzi G, Disalvatore D, Monturano M, Pece S, Viale G, Di Fiore PP, Boniolo G. A trust-based pact in research biobanks. From theory to practice. Bioethics. 2016;30(4):260-71. doi:10.1111/bioe. 12184 .

78. Golubnitschaja O, Lemke HU, Kapalla M, Kent A. Design in predictive, preventive and personalised medicine. In: Kuksa I, Fisher T, editors. Design for Personalisation. London: Gower Publishing; 2017. in press. 
79. Ausweger C, Burgschwaiger E, Kugler A, Schmidbauer R, Steinek I, Todorov Y, Thurnher D, Krapfenbauer K. Economic concerns about global healthcare in lung, head and neck cancer: meeting the economic challenge of predictive, preventive and personalized medicine. EPMA J. 2010;1(4):627-31. doi:10.1007/s13167-010-0054-x.

80. Brown PM. Personalized medicine and comparative effectiveness research in an era of fixed budgets. EPMA J. 2010;1 (4):633-40. doi:10.1007/s13167-010-0058-6.

81. Golubnitschaja O, Kinkorova J, Costigliola V. Predictive, preventive and personalised medicine as the hardcore of 'Horizon 2020': EPMA position paper. EPMA J. 2014;5(1):6. doi:10.1186/1878-5085-5-6.

82. Akhmetov I, Bubnov RV. Assessing value of innovative molecular diagnostic tests in the concept of predictive, preventive, and personalized medicine. EPMA J. 2015;6:19. doi:10.1186/s13167-015-0041-3.

\section{Submit your next manuscript to BioMed Central} and we will help you at every step:

- We accept pre-submission inquiries

- Our selector tool helps you to find the most relevant journal

- We provide round the clock customer support

- Convenient online submission

- Thorough peer review

- Inclusion in PubMed and all major indexing services

- Maximum visibility for your research

Submit your manuscript at www.biomedcentral.com/submit 